\title{
IL-7 abrogates memory $T$ regulatory cell functions by modulation of CD39/ATP axis in vitro and in vivo in HIV infected and non-infected patients
}

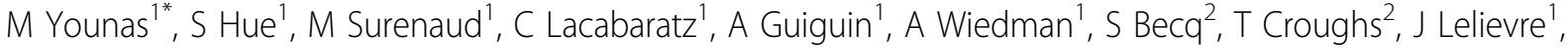 \\ Y Levy ${ }^{1}$
}

From AIDS Vaccine 2012

Boston, MA, USA. 9-12 September 2012

\section{Background}

IL-7 cytokine regulate the expansion and maturation of $\mathrm{T}$ cells. The good safety profile of IL-7 raises the opportunity of its use as a vaccine adjuvant. However, its role on the regulation of $\mathrm{T}$ cell responses has not been explored. We investigated the effects of IL-7 on regulatory $\mathrm{T}$ cell (Treg) functions invitro and invivo.

\section{Methods}

Treg and CD8+ T cells were isolated from healthy donors $(\mathrm{n}=10)(\mathrm{HD})$ and chronically HAART treated HIV+ pt enrolled in a phase I/II IL-7 INSPIRE study $(\mathrm{n}=6 \mathrm{X})$ (Levy et al, CID, in press). Treg subpopulations (naïve CD45RA+FoxP3++CD45RA+CD25++CD127+/-, memory (mTreg) Foxp3highCD45RA-CD25highCD127low, FoxP3++CD45RA-CD25++CD127+/-) were cultured with IL-7 (10 ng/ml). Phenotype (CD39, Bcl-2, Stat5P, purinergic receptor P2X7R), suppression of the proliferation of autologous antiCD3 activated CD8+ T cells (CFSE stained) and cytokine profile (IL-17 production) of Treg were analyzed.

\section{Results}

In HD, IL-7 induces expression of STAT5 and BCL-2 on all Treg populations. IL-7 reduces the suppressive effects of mTreg on CD8+ proliferation (\% CFSE low w/ wo IL7 was $15 \%$ and $40 \%$, respectively, $\mathrm{n}=4, \mathrm{P}=0.01$ ). This effect was associated with a down-modulation of CD39 enzyme (MFI 65 vs 90 w/wo IL7, $\mathrm{P}=0.01$ ) and an increase of P2X7R expression. IL-7 effect was reproduced using anti-CD39 blocking antibody and PPAD (inhibitor of P2X7R). IL-7 incubated Treg switched to a Th17 phenotype as assessed by the increase of Th17 production and RORgC expression. IL-7 treated patients exhibited a decrease of the frequency of Treg/CD39+ and an increase of RORgC mRNA in PBMCs as compared to pre-IL-7 therapy.

\section{Conclusion}

IL-7 relieves the suppressive effect of mTreg through a modulation of the CD39/ATP axis. By increasing P2X7R expression, IL-7 increases the susceptibility of these cells to ATP, a trigger of Th17 differentiation. An effect also observed in IL-7 treated patients. These results suggest that IL-7 could be used as adjuvant to reinforce T cell responses.

\section{Author details}

${ }^{1}$ Faculté de médecine, Université Paris-Est, Créteil, Créteil, France. ${ }^{2}$ Cytheris, France.

Published: 13 September 2012

\section{doi:10.1186/1742-4690-9-S2-P290}

Cite this article as: Younas et al:: IL-7 abrogates memory $\mathrm{T}$ regulatory cell functions by modulation of CD39/ATP axis in vitro and in vivo in HIV infected and non-infected patients. Retrovirology 2012 9(Suppl 2): P290.

${ }^{1}$ Faculté de médecine, Université Paris-Est, Créteil, Créteil, France

Full list of author information is available at the end of the article

(c) 2012 Younas et al; licensee BioMed Central Ltd. This is an Open Access article distributed under the terms of the Creative Commons 